\title{
A Mathematical Model on the Immune System Role in Achieving Better Outcomes of Cancer Chemotherapy
}

\author{
W.F.F.M. GIL ${ }^{1}$, T. CARVALHO ${ }^{2}$, P.F.A. MANCERA ${ }^{3}$ and \\ D.S. RODRIGUES ${ }^{4 *}$
}

Received on August 8, 2018 / Accepted on February 28, 2019

\begin{abstract}
The incidence of neoplastic diseases shows that the search for more effective cancer treatments is still necessary. In addition to standard treatments such as chemo- and radiotherapy, new treatment modalities have been focused in recent advances in immunology. Since little has been discussed about the biological implications of chemotherapy with respect to its impact on the immune system and on the other normal, "healthy" cells, we devote the present mathematical modeling work to do so. First, we prove the invariance of the region where all state variables remain positive (i.e., the number of cancer cells, normal cells and immune cells, and also the amount of chemotherapy). Afterward, we analyze the model in terms of the linear stability of the system and establish a necessary and sufficient condition for the local stability of the cure equilibrium point. Moreover, we simulate some scenarios involving both the immune system and chemotherapy, showing that a reasonable treatment strategy occurs when these are combined suitably.
\end{abstract}

Keywords: cancer, immune system, chemotherapy, mathematical modeling, ordinary differential equations.

\section{INTRODUCTION}

In the near future, cancer has the potential to become the leading cause of premature death, overshadowing cardiovascular diseases [15]. Classical treatments for cancer include chemotherapy, radiotherapy, and surgery. More recently, immunotherapy has been also an alternative to fight against cancer growth. Although there is a consistent understanding of the immunological responses to various pathogens, many aspects of the tumor-immune interaction remain unknown not only from the biological point of view but also quantitatively.

\footnotetext{
*Corresponding author: Diego S. Rodrigues - E-mail: diego.rodrigues@ft.unicamp.br - https://orcid.org/ 0000-0002-0016-1715

${ }^{1}$ UNESP, Programa de Pós-graduação em Biometria, 18618-689 Botucatu, Brazil. E-mail: wesmoragil@gmail.com

${ }^{2}$ USP, Departamento de Computação e Matemática, Faculdade de Filosofia, Ciências e Letras, 14040-901 Ribeirão Preto, Brazil. E-mail: tiagocarvalho@usp.br

${ }^{3}$ UNESP, Departamento de Bioestatística, Instituto de Biociências, 18618-689 Botucatu, Brazil. E-mail: paulo.mancera@unesp.br

${ }^{4}$ UNICAMP, Faculdade de Tecnologia, 13484-332 Limeira, Brazil. E-mail: diego.rodrigues@ft.unicamp.br
} 
Mathematical models of tumor growth under the influence of the immune system have been addressed in several papers by de Pillis and collaborators (e.g., $[4,5])$. In the latter, the authors make use of estimated parameters through experimental data to propose different ways of tumorimmune interaction and tumor dynamics models, which are further validated. With respect to tumor-immune interaction with chemotherapy, the search for better strategies of treatment is also addressed by de Pillis et al. in [2,3], where the authors report tests with different optimal control strategies for chemotherapy.

In the present paper, based on $[2,6,8]$ we propose a mathematical model that involves the influence of the immune system and chemotherapy on tumor cells, but also the normal cells and the unavoidable effect of chemotherapy on these. First, we show that the region of practical interest is invariant (once state variables are set positive they remain positive). Afterward, we analyze the model in terms of the linear stability of the system and establish a necessary and sufficient condition for the local stability of the cure equilibrium point. We also address some scenarios involving both the immune system and chemotherapy, showing that a reasonable treatment strategy occurs when these are combined suitably.

\section{MODEL}

Based on [6], [2] and [11], let us consider $N_{1}$ the number of tumor cells, $N_{2}$ the number of normal cells, $I$ the number of cells of the immune system, and $Q$ the amount of chemotherapeutic drug. Our proposed model is given by:

$$
\left\{\begin{array}{l}
\frac{d N_{1}}{d t}=r_{1} N_{1}\left(1-\frac{N_{1}}{k_{1}}-\frac{\alpha_{12} N_{2}}{k_{1}}\right)-c_{1} I N_{1}-\frac{\mu N_{1} Q}{a+Q} \\
\frac{d N_{2}}{d t}=r_{2}-\alpha_{21} N_{2} N_{1}-\frac{v N_{2} Q}{b+Q} \\
\frac{d I}{d t}=s-m I+\frac{\rho N_{1} I}{\gamma+N_{1}}-c_{2} N_{1} I-\frac{\delta I Q}{c+Q} \\
\frac{d Q}{d t}=q(t)-\lambda Q
\end{array}\right.
$$

Although there are different mathematical models that are used to describe tumor growth, we consider the logistic model (see [14]), where $r_{1}$ is the intrinsic growth rate, with the tumor cells carrying capacity given by $k_{1}$. The coefficients of competition between the populations $N_{1}$ and $N_{2}$ are given by $\alpha_{i j}$, which measure the effects of population $j$ on the population $i(i, j=1,2)$. The parameter $r_{2}$ represents the total constant reproduction of normal cells (see [6]).

The dynamics in the immune cell population is activated by the tumor population at a rate $\rho, \gamma$ being the half-saturation constant of the Michaelis-Menten functional response given by $\rho I N_{1} /\left(\gamma+N_{1}\right)$ (see [2]), and also there is a natural death rate of immune cells given by $m$. As we know that some immune cells can directly eliminate tumor cells (see [2]), two more terms are in order, $-c_{1} I N_{1}$ and $-c_{2} I N_{1}$, the latter representing the inactivation of immune cells acting 
on tumor cells and the former is due to the death of tumor cells due to the action of the immune system. The term $s$ describes a natural source of immune cells (see [8]).

In order to model the amount of chemotherapy injected in the system, the function $q=q(t)$ models the infusion of the drug into the system and $\lambda$ is the drug washout rate. As in [12], the response of each cell population to the chemotherapy is considered to be of a Michaelis-Menten form, with half-saturation parameters $a, b$ and $c ; \mu$ is the treatment rate of the tumor cells; $v$ is the mortality rate of normal cells due to treatment; and $\delta$ represents the mortality rate of immune cells due to the chemotherapeutic drug (see also [13]).

Throughout the paper, the drug administration $q(t)$ takes two different forms:

1. Continuous administration

$q(t)=q>0$, and the drug infusion is at a constant rate.

2. Administration in cycles

Following [9], $q(t)$ is a periodic function defined as

$$
q(t)=\left\{\begin{array}{l}
q_{\mathrm{p}}>0, \quad n<t \leq n+\tau \\
0, \quad n+\tau<t \leq n+T
\end{array}\right.
$$

where $T$ is the time between drug infusions, $n=0, T, 2 T, 3 T, \cdots, m T$ represents the instants of administration, and $\tau$ is the time taken for infusion.

\section{RESULTS}

We analyze the stability of the system firstly considering that there is no chemotherapy and then we address the chemotherapeutic case.

\subsection{Stability analysis without chemotherapy}

As the model represents the time evolution of non-negative state variables, it is desirable that these variables remain with this property. But even for a positive initial condition, there is no apparent reason for the solutions not to converge to an equilibrium with one or more negative coordinates. However, from this point on we will prove that this cannot occur given the dynamics on the planes $N_{1}, N_{2}$ and $I$.

- On the plane $N_{2}=0$ the model is given by:

$$
\left\{\begin{array}{l}
\frac{d N_{1}}{d t}=r_{1} N_{1}\left(1-\frac{N_{1}}{k_{1}}\right)-c_{1} I N_{1} \\
\frac{d N_{2}}{d t}=r_{2} \\
\frac{d I}{d t}=s-m I+\frac{\rho N_{1} I}{\gamma+N_{1}}-c_{2} N_{1} I
\end{array}\right.
$$


As $d N_{2} / d t=r_{2}>0$ the normal cell population is strictly increasing, that is, the trajectories will follow the direction from $N_{2}<0$ to $N_{2}>0$. Then, if the initial condition comprises $N_{2}(0) \geq 0$, then the values of $N_{2}(t)$, with $t>0$, will always be positive (see Figure 1).

- On the plane $I=0$ the system is written as:

$$
\left\{\begin{array}{l}
\frac{d N_{1}}{d t}=r_{1} N_{1}\left(1-\frac{N_{1}}{k_{1}}-\frac{\alpha_{12} N_{2}}{k_{1}}\right) \\
\frac{d N_{2}}{d t}=r_{2}-\alpha_{21} N_{2} N_{1} \\
\frac{d I}{d t}=s
\end{array}\right.
$$

As $d I / d t=s>0$ the immune cells increase, that is, any trajectory will follow the direction from $I<0$ to $I>0$ (see Figure 2).

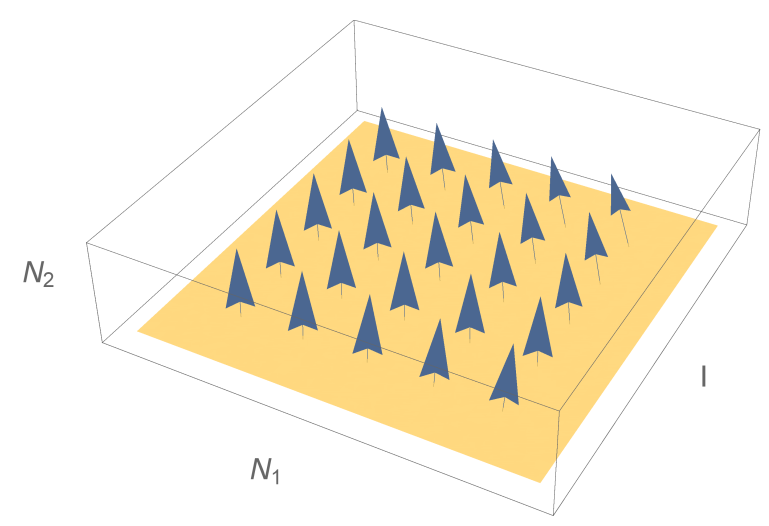

Figure 1: Vector field on the plane $N_{2}=0$.

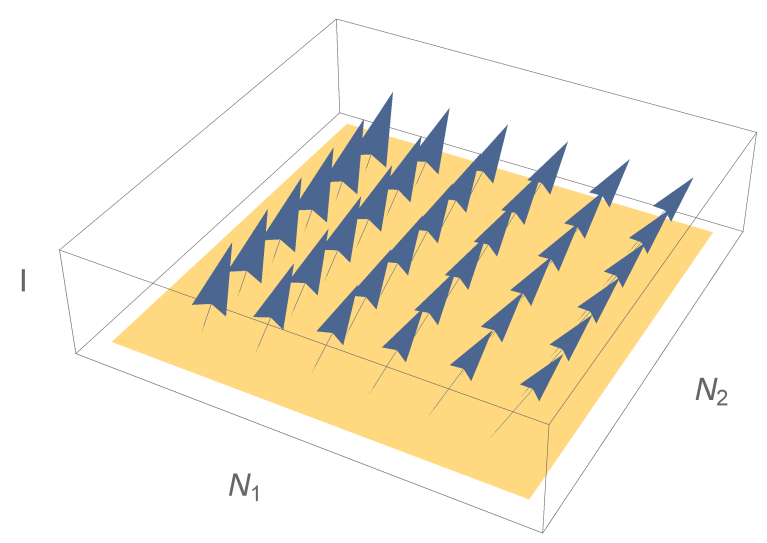

Figure 2: Vector field on the plane $I=0$. 
- When $N_{1}=0$ one gets the following system:

$$
\left\{\begin{array}{l}
\frac{d N_{1}}{d t}=0 \\
\frac{d N_{2}}{d t}=r_{2} \\
\frac{d I}{d t}=s-m I=f(I)
\end{array}\right.
$$

Note that this plane is an invariant subspace, since $d N_{1} / d t=0$. As a consequence, given an initial condition in which $N_{1}(0)=0$, the cancer population will always be zero. Moreover, solving the equations on this plane with initial condition $\left(0, N_{20}, I_{0}\right)$ one gets:

$$
\left\{\begin{array}{l}
N_{1}(t)=0 \\
N_{2}(t)=r_{2} t+N_{20} \\
I(t)=I_{0} e^{-m t}+\frac{s}{m}\left(1-e^{-m t}\right)
\end{array}\right.
$$

Considering the immune cells variation given by the third equation in (3.1), one sees that the straight line $H(t)=\left(N_{1}(t), N_{2}(t), I(t)\right)=\left(0, r_{2} t+N_{2}(0), s / m\right)$ is an invariant set inside the plane $N_{1}=0$. Moreover, since $\partial f / \partial I=-m<0$, where $f(I)=s-m I$, we conclude that $H(t)$ is an attractor, which means that for a large value of $t>0$ the population $I$ converges to $s / m$ and the population $N_{2}$ goes to infinity (see Figure 3 ). These subspaces are very illustrative because they are related to the elimination of cancer cells.

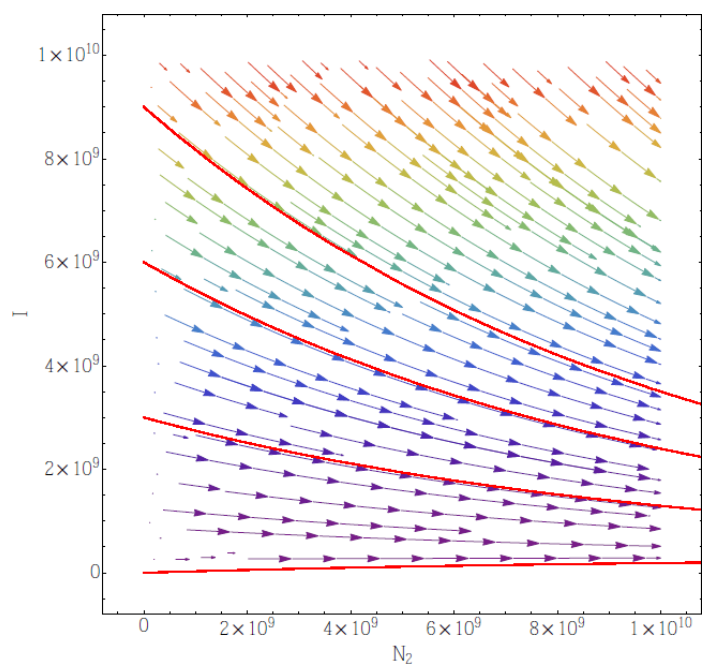

Figure 3: Phase portrait on the plane $N_{1}=0$, with parameters listed in Table 1. 
Table 1: List of parameters for numerical solutions of the model (2.1).

\begin{tabular}{ccc}
\hline Parameters & Values & Unity \\
\hline$r_{1}$ & $10^{-2}$ & day $^{-1}$ \\
$k_{1}$ & $10^{12}$ & cells \\
$\alpha_{12}$ & $9 \times 10^{-5}$ & - \\
$c_{1}$ & $5 \times 10^{-11}$ & cells $^{-1}$ day $^{-1}$ \\
$\mu$ & 8 & day $^{-1}$ \\
$a$ & $2 \times 10^{3}$ & mg \\
$r_{2}$ & $10^{7}$ & cells day $^{-1}$ \\
$\alpha_{21}$ & $9 \times 10^{-16}$ & cells day $^{-1}$ \\
$v$ & 8 & day $^{-1}$ \\
$b$ & $5 \times 10^{6}$ & mg $^{-1}$ \\
$s$ & $3 \times 10^{5}$ & cells day $^{-1}$ \\
$m$ & $10^{-3}$ & day $^{-1}$ \\
$\rho$ & $10^{-12}$ & day $^{-1}$ \\
$\gamma$ & $10^{2}$ & cells $^{-12}$ \\
$c_{2}$ & $10^{-13}$ & cells $^{-1}$ day $^{-1}$ \\
$\delta$ & $10^{4}$ & day $^{-1}$ \\
$c$ & $5 \times 10^{6}$ & mg $^{-1}$ \\
$\lambda$ & 4.16 & day $^{-1}$ \\
\hline
\end{tabular}

\subsection{Stability analysis with chemotherapy}

Now we add chemotherapy in our analysis, in order to study the full model (2.1). We have the same behavior in the subspaces $N_{2}=0$ and $I=0$, as the situation without chemotherapy. Let $N_{1}=0$ and $q(t)=q$ (constant) results in the following system:

$$
\left\{\begin{array}{l}
\frac{d N_{1}}{d t}=0 \\
\frac{d N_{2}}{d t}=r_{2}-\frac{v N_{2} Q}{b+Q} \\
\frac{d I}{d t}=s-m I-\frac{\delta I Q}{c+Q} \\
\frac{d Q}{d t}=q-\lambda Q=h(Q)
\end{array}\right.
$$

The subspace is still invariant, since to the initial condition $\left(N_{1}, N_{2}, I, Q\right)=\left(0, N_{20}, I_{0}, Q_{0}\right)$ one gets

$$
Q(t)=Q_{0} e^{-\lambda t}+\frac{q}{\lambda}\left(1-e^{-\lambda t}\right)
$$


Since $N_{1}=0$ is the solution to the cancer cells population, then we write $g(t)$ and $f(t)$ as the solutions to the normal and immune cells populations, respectively. Let us consider the solution of the system of equations given by $Y(t)=\left(N_{1}(t), N_{2}(t), I(t), Q(t)\right)$ and $W$, the subspace where $N_{1}=0$. For an initial condition $P=\left(0, N_{2}(0), I(0), Q(0)\right) \in W$, the solution is given by

$$
Y(t)=\left(0, g(t), f(t), Q(0) e^{-\lambda t}+\frac{q}{\lambda}\left(1-e^{-\lambda t}\right)\right) \in W
$$

Then, if the solution is in this subspace, it will remain there, resulting that $W$ is invariant. Consequently, we can discard $N_{1}<0$.

The equilibrium point of the chemotherapeutic equation is given by $Q(t)=q / \lambda$ and it is stable, since $\partial h / \partial Q=-\lambda$, where $h(Q)=q-\lambda Q$. We have another invariant subspace $W_{3} \subset W$, given by $N_{1}=0$ and $Q=q / \lambda$. It is invariant because to $P=\left(0, N_{2}(0), I(0), q / \lambda\right) \in W_{3}$, the solution given by $Y(t)=(0, g(t), f(t), q / \lambda) \in W_{3}$. When $q=0$ and $Q(0)=0$, we conclude that $W_{3}$ has a similar behavior as the invariant plane without chemotherapy, where the solutions are getting closer to the invariant line $I=s / m$ and $N_{2} \rightarrow \infty$. As $q$ increases, $q>0$, there is an equilibrium point on the invariant line with coordinates

$$
P_{\mathrm{inf}}=\left(N_{2}, I, Q\right)=\left(\frac{(b \lambda+q) r_{2}}{q v}, \frac{s(q+c \lambda)}{m q+q \delta+c m \lambda}, \frac{q}{\lambda}\right)
$$

on the subspace with $N_{1}=0$. As $N_{1}$ does not change on the invariant subspace $W$ in $3 \mathrm{D}$, we analyze the stability on this subspace. The Jacobian matrix is:

$$
J_{1}=\left[\begin{array}{ccc}
-\frac{v Q}{b+Q} & 0 & \frac{v N_{2} Q}{(b+Q)^{2}}-\frac{v N_{2}}{b+Q} \\
0 & -m-\frac{\delta Q}{c+Q} & \frac{\delta I Q}{(c+Q)^{2}}-\frac{\delta I}{c+Q} \\
0 & 0 & \lambda
\end{array}\right]
$$

Evaluating the Jacobian matrix on $P_{\text {inf }}$ it follows:

$$
J_{1}\left(P_{\text {inf }}\right)=\left[\begin{array}{ccc}
-\frac{v q}{b \lambda+q} & 0 & -\frac{b r_{2} \lambda^{2}}{q^{2}+b q \lambda} \\
0 & -m-\frac{\delta q}{c \lambda+q} & -\frac{c s \delta \lambda^{2}}{(q+c \lambda)(q(m+\delta)+c m \lambda)} \\
0 & 0 & \lambda
\end{array}\right]
$$

The eigenvalues of (3.2) are given by

$$
\xi_{1}=-\lambda<0,
$$




$$
\xi_{2}=-\left(\frac{q v}{b \lambda+q}\right)<0
$$

and

$$
\xi_{3}=-\left(\frac{m c \lambda+m q+q \delta}{c \lambda+q}\right)<0,
$$

implying that the equilibrium point is stable on this subspace. This point, $P_{\text {inf }}$, occurs due to the killing of normal cells by the chemotherapeutic drug.

Now we study the cancer model in the full domain. The system (2.1) has five equilibrium points for $q>0$; four of them have the following structure

$$
P^{*}=\left(N_{1}^{*}, N_{2}^{*}, I^{*}, \frac{q}{\lambda}\right)
$$

and the other is

$$
P_{\mathrm{inf}}=\left(0, \frac{r_{2}(q+b \lambda)}{q v}, \frac{q s+c s \lambda}{m q+q \delta+c m \lambda}, \frac{q}{\lambda}\right)
$$

The point $P_{\text {inf }}$ represents the extinction of cancer cells, and it was analyzed in the subspace $W$, with $Q=q / \lambda>0$. The Jacobian matrix is

$$
J_{2}=\left[\begin{array}{cccc}
\bar{A} & -\frac{\alpha_{12} N_{1} r_{1}}{k_{1}} & -c_{1} N_{1} & \frac{\mu N_{1} Q}{(a+Q)^{2}}-\frac{\mu N_{1}}{a+Q} \\
-\alpha_{21} N_{2} & -\alpha_{21} N_{1}-\frac{v Q}{b+Q} & 0 & \frac{v N_{2} Q}{(b+Q)^{2}}-\frac{v N_{2}}{b+Q} \\
\bar{B} & 0 & \bar{C} & \frac{\delta I Q}{(c+Q)^{2}}-\frac{\delta I}{c+Q} \\
0 & 0 & 0 & \lambda
\end{array}\right]
$$

where

$$
\begin{gathered}
\bar{A}=-c_{1} I-\frac{N_{1} r_{1}}{k_{1}}+r_{1}\left(1-\frac{N_{1}}{k_{1}}-\frac{\alpha_{12} N_{2}}{k_{1}}\right)-\frac{\mu Q}{a+Q}, \\
\bar{B}=-c_{2} I-\frac{I N_{1} \rho}{\left(N_{1}+\gamma\right)^{2}}+\frac{I \rho}{\left(N_{1}+\gamma\right)},
\end{gathered}
$$

and

$$
\bar{C}=-m-c_{2} N_{1}-\frac{\delta Q}{c+Q}+\frac{N_{1} \rho}{\left(N_{1}+\gamma\right)}
$$


Consequently, $J_{2}\left(P_{\text {inf }}\right)$ takes the form:

$$
J_{2}\left(P_{\text {inf }}\right)=\left[\begin{array}{cccc}
\bar{D} & 0 & 0 & 0 \\
-\frac{\alpha_{21} r_{2}(q+b \lambda)}{q v} & -\frac{v q}{b+b \lambda} & 0 & -\frac{b r_{2} \lambda^{2}}{q^{2}+b q \lambda} \\
-\frac{s(q+c \lambda)\left(c_{2} \gamma-\rho\right)}{\gamma(q \delta+m)(q+c \lambda)} & 0 & -\frac{m q+q \delta+c m \lambda}{q+c \lambda} & \bar{E} \\
0 & 0 & 0 & \lambda
\end{array}\right]
$$

where

$$
\bar{D}=-\frac{c_{1} s(q+c \lambda)}{q \delta+m(q+c \lambda)}-\frac{q \mu}{q+a \lambda}+r_{1}-\frac{r_{1} \alpha_{12} r_{2}(q+b \lambda)}{k_{1} q v}
$$

and

$$
\bar{E}=-\frac{c s \delta \lambda^{2}}{(q+c \lambda)(q \delta+m(q+c \lambda))}
$$

The eigenvalues of this matrix are

$$
\begin{gathered}
\chi_{1}=-\lambda, \\
\chi_{2}=-\left(\frac{q v}{q+b \lambda}\right), \\
\chi_{3}=-\left(\frac{m q+q \delta+c m \lambda}{q+c \lambda}\right),
\end{gathered}
$$

and

$$
\chi_{4}=r_{1}-\frac{c_{1} s(c \lambda+q)}{m c \lambda+m q+\delta q}-\frac{\mu q}{a \lambda+q}-\frac{\alpha r_{2}(b+q)}{v q}
$$

The first three eigenvalues are negative since the parameters are positive. The fourth one will be negative under the following condition:

$$
r_{1}<\frac{c_{1} s(c \lambda+q)}{m c \lambda+m q+\delta q}+\frac{\mu q}{a \lambda+q}+\frac{\alpha r_{2}(b+q)}{v q}
$$

\subsection{Numerical simulations: chemotherapy in cycles}

To set the chemotherapeutic schedule, we define the function $q$ based on a drug regimen already used in practice. In the specific case of chronic lymphocytic leukemia (CLL), one possible choice is a drug combination with pentostatin, cyclophosphamide and rituximab, usually known as PCR protocol [7]. In our numerical simulations, however, we only consider the use of cyclophosphamide [1].

The chemotherapeutic regimen consists of a 21-day, 5- or 6-cycle schedule of $600 \mathrm{mg}$ of cyclophosphamide per $\mathrm{m}^{2}$ of body surface per infusion. For a hypothetical human patient of height $1.70 \mathrm{~m}$ and weight $65 \mathrm{~kg}$, resulting in a body surface of $1.75 \mathrm{~m}^{2}$ [10], the dose per infusion is 
$600 \times 1.75=1050 \mathrm{mg}$. We consider a drug infusion time of 3 hours or $1 / 8$ day, resulting in $q_{\mathrm{p}}=600 \times 1.75 /(1 / 8)=8400 \mathrm{mg} /$ day. The other parameters are listed in Table 1.

Then

$$
q(t)=\left\{\begin{array}{c}
8400, n \leq t<n+1 / 8 \\
0, n+1 / 8 \leq t<n+21
\end{array}\right.
$$

with $n=0,21,42,63,84$ for 5 infusions or $n=0,21,42,63,84,105$ for 6 infusions.

We have simulated two situations, shown in Figure 4, one with $s=3 \times 10^{5}$ and another with $s=7 \times 10^{5}$. We observe how important is the value of $s$ (how efficient the immune system is), resulting in the cure or disease relapse.

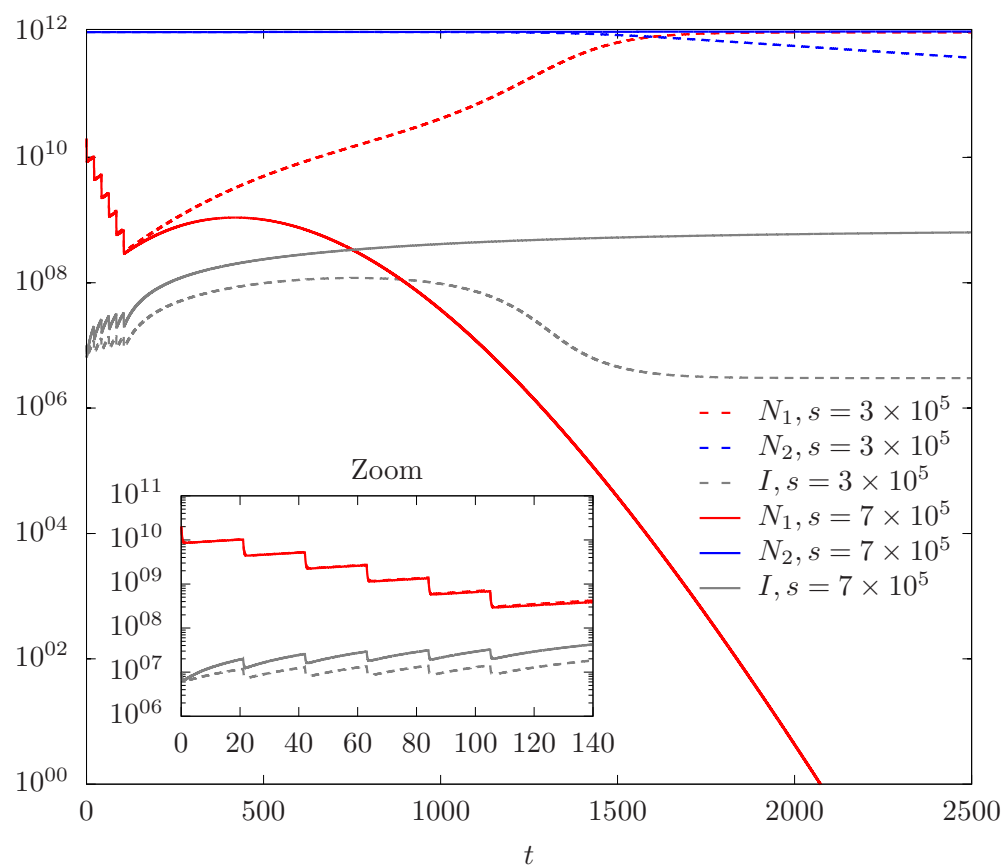

Figure 4: Tumoral dynamics with $s=3 \times 10^{5}$ and $s=7 \times 10^{5}$. Initial condition: $N_{1}(0)=2 \times 10^{10}$, $N_{2}(0)=10^{12}, I(0)=10^{7}$ and $Q=0$.

In Figure 5 we have taken $s=4 \times 10^{5}$ and the number of infusions 5 or 6 . We observe that one more infusion results in a change of the equilibrium point attained, from the failure of treatment (5 infusions) to cure (6 infusions). 


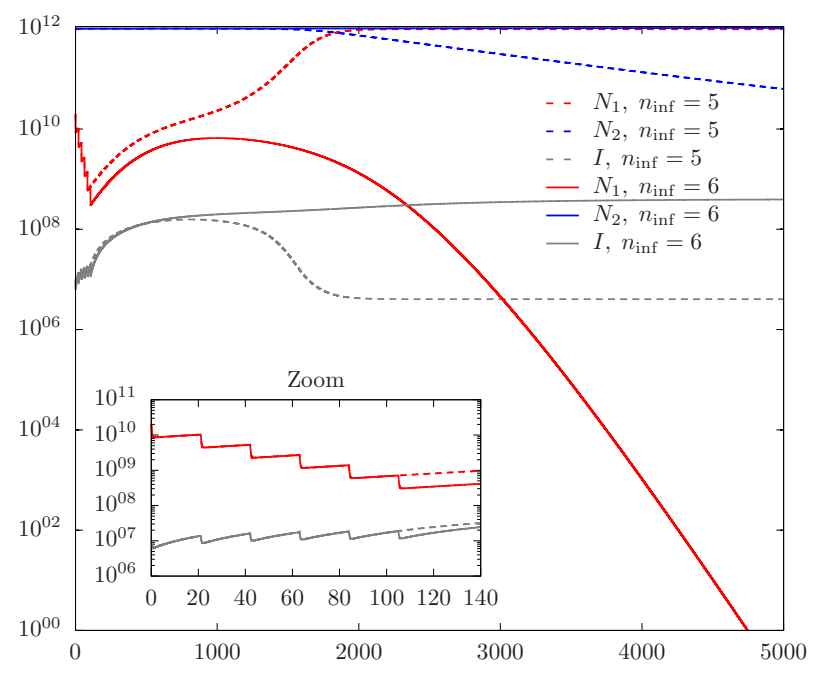

Figure 5: Tumoral dynamics with $s=4 \times 10^{5}$ and 5 or 6 infusions. Initial condition: $N_{1}(0)=$ $2 \times 10^{10}, N_{2}(0)=10^{12}, I(0)=10^{7}$ and $Q(0)=0$.

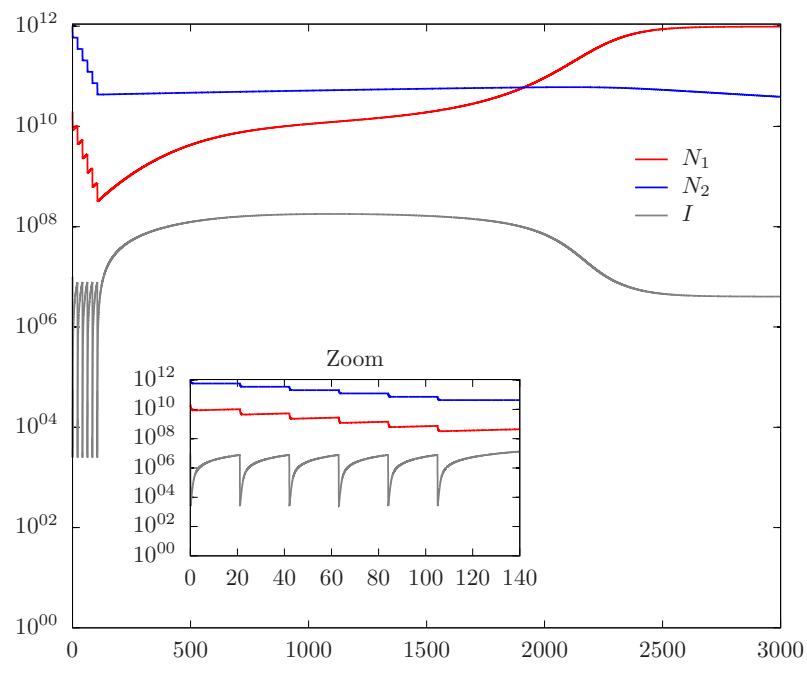

Figure 6: Cells population dynamics with $s=4 \times 10^{5}, \delta=10^{6}$ and $v=10^{3}$. Initial condition: $N_{1}(0)=2 \times 10^{10}, N_{2}(0)=10^{12}, I(0)=10^{7}$ and $Q(0)=0$.

Figure 6 illustrates the cytotoxic effects of the chemotherapeutic drug on the cells population. We have set new values for the parameters $\delta$ and $v: \delta=10^{6}$ and $v=10^{3}$. We note that the cure is not anymore possible and the number of normal cells decreases. The action of the immune system is limited by chemotherapy. 
We have increased the value of $\rho$, which is the rate of production of immune cells stimulated by the cancer cells. Keeping $s=4 \times 10^{5}$ and with 5 or 6 infusions, the number of cancer cells does not go to zero as it can be seen in Figure 7. In this situation, the cancer growth is postponed, but not avoided (see Figure 8).

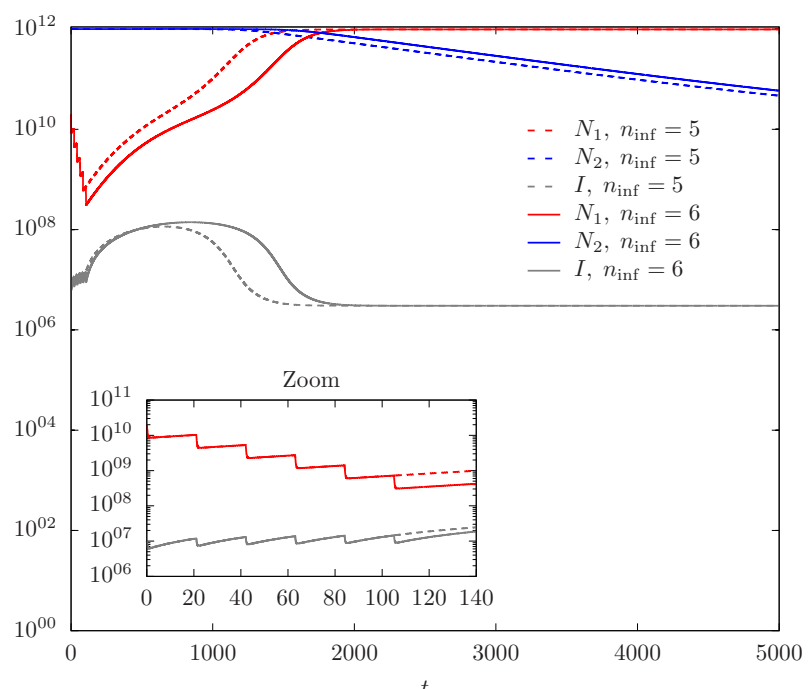

Figure 7: Tumor and immune cells dynamics with $\rho=4 \times 10^{4}$ and 5 or 6 infusions. Initial condition: $N_{1}(0)=2 \times 10^{10}, N_{2}(0)=10^{12}, I(0)=10^{7}$ and $Q(0)=0$.

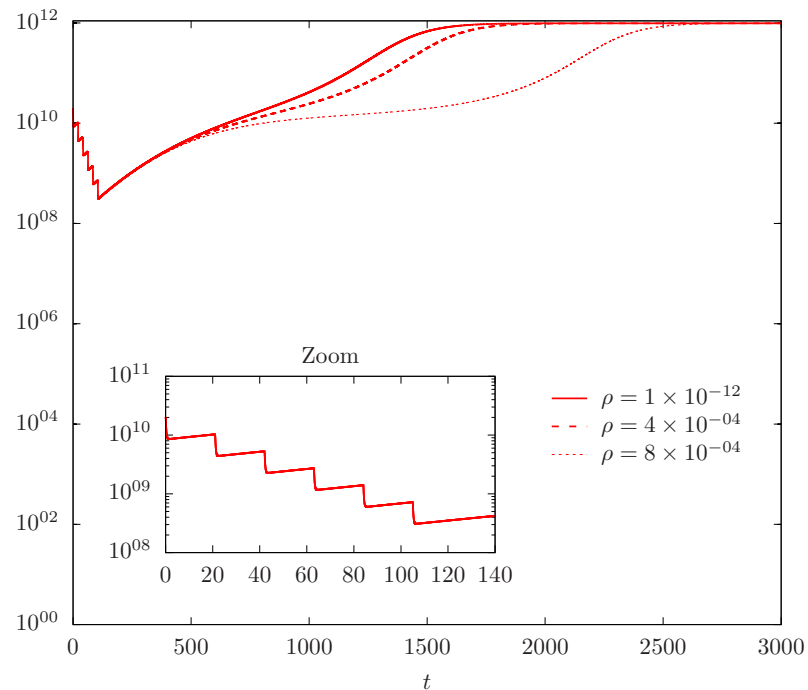

Figure 8: Tumor dynamics to three different values of $\rho$. Initial condition: $N_{1}(0)=2 \times 10^{10}$, $N_{2}(0)=10^{12}, I(0)=10^{7}$ and $Q(0)=0$. 
To show the importance of the immune system, we have fixed $\rho$ as $\rho=4 \times 10^{4}$ and chosen $s=4 \times 10^{5}$, with 5 infusions of the drug. The cancer cells go to zero, even with 5 infusions of the chemotherapeutic drug, as seen in Figure 9. This is a crucial situation since we are achieving the cure administrating a lesser amount of chemotherapy.

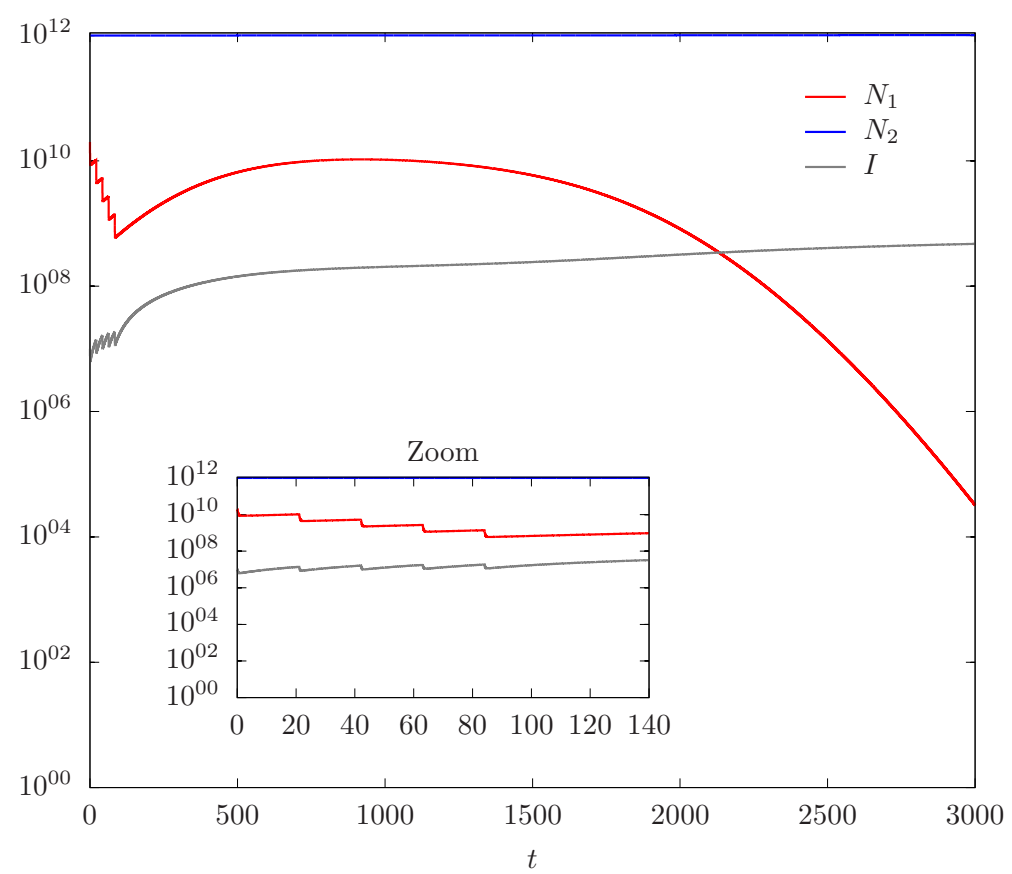

Figure 9: Cells population dynamics with $\rho=4 \times 10^{4}$ and $s=4 \times 10^{5}$. Initial condition: $N_{1}(0)=$ $2 \times 10^{10}, N_{2}(0)=10^{12}, I(0)=10^{7}$ and $Q(0)=0$.

\section{CONCLUSIONS}

We developed an ODE mathematical model for tumor growth under the action of the immune system and chemotherapy. We established the positivity of the dependent variables of the model. As a result, we presented the stability analysis of the referred system, revealing the condition that must hold for the cure equilibrium to be attained. From the numerical simulations, we have shown both immune system and chemotherapy are important to the decreasing of tumor population, but a very strong immune system combined with chemotherapy is much more crucial to reducing the number of tumor cells to a low level. According to our results, it is also crucial to allow the immune system to recover from the chemotherapy before considering more eventual applications of oncological therapies. As a final remark, as it is shown in Figures 8 and 9, let us note that the success of chemotherapy critically depends on the intensity in which the tumor cells stimulate the immune cell production. In the near future, this could be explored in detail in some immunotherapeutic strategy. 
RESUMO. A incidência de doenças neoplásicas mostra que a busca por tratamentos mais eficazes contra o câncer ainda é necessária. Além dos tratamentos padrão, como quimioterapia e radioterapia, novas modalidades de tratamento têm sido focadas nos recentes avanços na imunologia. Como pouco tem sido discutido sobre as implicações biológicas da quimioterapia em relação ao seu impacto tanto no sistema imunológico como em outras células normais e "saudáveis", dedicamos o presente trabalho de modelagem matemática a fazê-lo. Primeiro, provamos a invariância da região na qual todas as variáveis de estado permanecem positivas (ou seja, o número de células cancerosas, células normais e células imunes, e também a quantidade de quimioterapia). Em seguida, analisamos o modelo em termos de estabilidade linear do sistema e estabelecemos uma condição necessária e suficiente para a estabilidade local do ponto de equilíbrio de cura. Além disso, simulamos alguns cenários envolvendo tanto o sistema imunológico quanto a quimioterapia, mostrando que uma estratégia de tratamento razoável ocorre quando estes são combinados adequadamente.

Palavras-chave: câncer, sistema imune, quimioterapia, modelagem matemática, equações diferenciais ordinárias.

\section{REFERENCES}

[1] F.T. Awan. Cure for CLL? Blood, 127(3) (2016), 274 p. doi:10.1182/blood-2015-11-678532. URL http://www . bloodjournal .org/content/127/3/274.

[2] G.L. De Pillis \& A. Radunskaya. A mathematical tumour model with immune resistance and drug therapy: An optimal control approach. Journal of Theoretical Medicine, 3(2) (2001), 79-100. doi:10.1080/ 10273660108833067. URL https://www.hindawi.com/journals/cmmm/2001/318436/abs/.

[3] L.G. de Pillis, W. Gu, K.R. Fister, T. Head, K. Maples, A. Murugan, T. Neal \& K. Yoshida. Chemotherapy for tumors: An analysis of the dynamics and a study of quadratic and linear optimal controls. Mathematical Biosciences, 209(1) (2007), 292-315. doi:10.1016/j.mbs.2006.05.003. URL http://www.sciencedirect.com/science/article/pii/S0025556406000836.

[4] L.G. de Pillis \& A. Radunskaya. A mathematical model of immune response to tumor invasion. In K.J. Bathe (editor), "Computational Fluid and Solid Mechanics 2003". Elsevier Science Ltd, Oxford (2003), pp. 1661-1668. doi:10.1016/B978-008044046-0.50404-8. URL https://www . sciencedirect.com/science/article/pii/B9780080440460504048.

[5] L.G. de Pillis, A.E. Radunskaya \& C.L. Wiseman. A validated mathematical model of cell-mediated immune response to tumor growth. Cancer Research, 65(17) (2005), 7950-7958. doi:10.1158/ 0008-5472.CAN-05-0564.

[6] A.C. Fassoni. "Modelos matemáticos em câncer abordando fase inicial e tratamento de tumor avascular (in portuguese)". Ph.D. thesis, Universidade Estadual de Campinas, Campinas (2016). URL http://repositorio.unicamp.br/jspui/handle/REPOSIP/307199.

[7] N.E. Kay, S.M. Geyer, T.G. Call, T.D. Shanafelt, C.S. Zent, D.F. Jelinek, R. Tschumper, N.D. Bone, G.W. Dewald, T.S. Lin, N.A. Heerema, L. Smith, M.R. Grever \& J.C. Byrd. Combination chemoimmunotherapy with pentostatin, cyclophosphamide, and rituximab shows significant clinical activity 
with low accompanying toxicity in previously untreated B chronic lymphocytic leukemia. Blood, 109(2) (2007), 405-411. doi:10.1182/blood-2006-07-033274. URL http://www. bloodjournal. org/content/109/2/405.

[8] V.A. Kuznetsov, I.A. Makalkin, M.A. Taylor \& A.S. Perelson. Nonlinear dynamics of immunogenic tumors: Parameter estimation and global bifurcation analysis. Bulletin of Mathematical Biology, 56(2) (1994), 295-321. doi:10.1016/S0092-8240(05)80260-5. URL http://www . sciencedirect. com/ science/article/pii/S0092824005802605.

[9] R. Martin \& K.L. Teo. "Optimal Control of Drug Administration in Cancer Chemotherapy". World Scientific Publishing Company, Singapore (1993). URL https://www.worldscientific.com/ worldscibooks/10.1142/2048.

[10] R.D. Mosteller. Simplified Calculation of Body-Surface Area. New England Journal of Medicine, 317(17) (1987), 1098. doi:10.1056/NEJM198710223171717. URL https://doi.org/10.1056/ NEJM198710223171717.

[11] D.S. Rodrigues, P.F. de Arruda Mancera, T. de Carvalho \& L.F. Gonçalves. A mathematical model for chemoimmunotherapy of chronic lymphocytic leukemia. Applied Mathematics and Computation, 349 (2019), 118-133. doi:10.1016/j.amc.2018.12.008. URL https://www.sciencedirect.com/ science/article/pii/S009630031831049X.

[12] D.S. Rodrigues \& P.F.A. Mancera. Mathematical analysis and simulations involving chemotherapy and surgery on large human tumours under a suitable cell-kill functional response. Mathematical Biosciences \& Engineering, 10 (2013), 221-234. doi:10.3934/mbe.2013.10.221. URL http: //aimsciences.org/article/doi/10.3934/mbe.2013.10.221.

[13] D.S. Rodrigues, P.F.A. Mancera \& S.T.R. Pinho. Understanding the antiangiogenic effect of metronomic chemotherapy through a simple mathematical model. Physica A: Statistical Mechanics and its Applications, 464 (2016), 251-266. doi:10.1016/j.physa.2016.07.076. URL http://www. sciencedirect.com/science/article/pii/S0378437116305167.

[14] V.G. Vaidya \& F.J. Alexandro Jr. Evaluation of some mathematical models for tumor growth. International Journal of Bio-Medical Computing, 13(1) (1982), 19-35. doi:10.1016/0020-7101(82)90048-4. URL http://www . sciencedirect.com/science/article/pii/0020710182900484.

[15] World Health Organization. Cancer. URL http://www.who.int/topics/cancer/en/. Http://www.who.int/topics/cancer/en/. Accessed: February 25, 2019. 